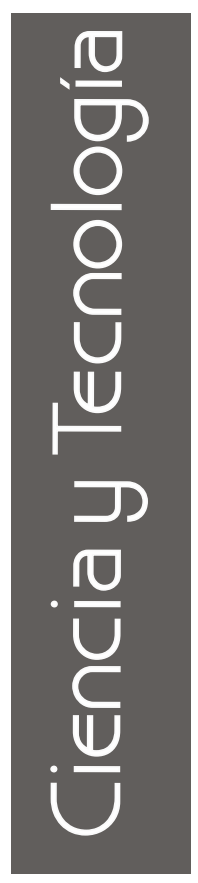

\title{
La Planificación Jerárquica y su Aplicación a la Cosecha de la Caña de Azúcar en Colombia
}

\author{
Irene Tischer* \\ Andrés Carrión**
}

* Ph.D. Profesora Asociada - Escuela de Ingeniería de Sistemas y Computación - Facultad de Ingeniería Universidad del Valle - Santiago de Cali.

E-mail:irene@eisc.univalle.edu.co

* * Ph.D. Profesor Catedrático - Departamento de Estadística e Investigación Operativa - Universidad Politécnica de Valencia - España.

E-mail:acarrion@eio.upv.es

Fecha de recepción: Octubre 16 de 2002

Fecha de aprobación: Marzo 26 de 2003

\section{RESUMEN}

En el presente trabajo se introducen los conceptos generales de la planificación jerárquica, enfoque usado para desarrollar procesos decisorios complejos. Se muestra la aplicación de la planificación jerárquica a la industria azucarera colombiana y su subsistema más complejo: la cosecha de la caña. Se propone un modelo de planificación jerárquica de la cosecha que considera los niveles estratégico, táctico y operativo y la integración entre ellos. En cada nivel se especifican los objetivos, el horizonte de planificación y los modelos cuantitativos que apoyan las decisiones. 
Construyendo un modelo de simulación, representativo para un sistema productivo azucarero, se valida la eficiencia del proceso decisorio propuesto y la compatibilidad entre los modelos. Los resultados de la simulación permiten concluir que al aplicar el modelo propuesto se aumenta la productividad del sector azucarero colombiano.

Palabras Clave: Industria azucarera, Planificación jerárquica, Modelos cuantitativos, Indicadores, Sistemas de soporte a las decisiones.

\section{ABSTRACT}

The present work introduces the general concepts of hierarchal planning, used to develop complex decision processes. We apply hierarchical planning to the Colombian sugar industry and its most complex subsystem: the sugar cane harvesting. We propose a hierarchical planning model for the sugar cane harvest that considers a strategic, tactic and operative level and integration between levels. For each level we specify the planning objectives and horizons and the quantitative models to support decisions. Constructing a simulation model, representative for the sugar production system, we validate the efficiency of the proposed decision process and the compatibility between the models in different decision levels. The simulation results permit to conclude that the application of the proposed model augments the productivity of the Colombian sugar industry.

Key Words: Sugar industry, Hierarchical planning, Quantitative models, Indicators, Decision support systems.

\section{LA PLANIFICACIÓN JERÁRQUICA}

\section{Conceptos:}

El uso de modelos cuantitativos en la toma de decisiones asegura la objetividad en la evaluación de alternativas y permite deducir en forma cuantitativa y abstracta la mejor solución a un problema dado. Sin embargo, por la complejidad creciente de los sistemas productivos, se vuelve siempre más difícil describir todos sus aspectos con un solo modelo. Un tal modelo monolítico debería incluir un gran número de variables, resultando difícil su solución e interpretación.

Por eso se usa generalmente una serie de modelos individuales para resolver problemas específicos, lo que consecuentemente conduce a la toma de decisiones en forma aislada para un subsistema. Esta solución no favorece necesariamente a la organización como un todo, llegando a ocurrir a veces que la decisión que mejora el subsistema, implica un empeoramiento del desempeño global de la organización.

En la organización se requiere ambos: Por un lado una visión general que permita conocer a grandes rasgos las tendencias que se presentan en el sistema en consideración, explorar nuevas alternativas de solución a los problemas y seleccionar entre ellas, las que más apropiadas parecen ser en el contexto considerado. Por otro lado, suficiente detalle para resolver los problemas tácticos y operativos.

La planificación jerárquica permite tomar decisiones basadas en información global en el nivel estratégico y detallar más en el momento de buscar apoyo en decisiones que abarcan aspectos parciales del sistema.

Por tratarse de una jerarquía decisoria y no de una serie de decisiones aisladas, se garantiza la compatibilidad entre las decisiones de los diferentes subsistemas, asegurando que en las decisiones detalladas no se pierde la visión general. Por otro lado, el uso de información agregada posibilita enfocarse en las tendencias globales (Hax et al. [6]; Anäxter [1]; Schneeweiss [7]). Los conceptos generales de la planificación jerárquica nos han llevado a la 
convicción de que esta estructura decisoria es la más apropiada para la planificación de la cosecha de la caña:

- La planificación jerárquica considera una jerarquía de niveles decisorios, orientada en la estructura organizacional del ingenio.

- Respeta los objetivos de cada nivel y garantiza la compatibilidad entre las decisiones en los diferentes niveles.

- Permite considerar diferentes horizontes de tiempo (largo, medio y corto plazo).

- Determina la información relevante para la toma de decisiones para cada nivel decisorio.

- Define para cada nivel decisorio los modelos que apoyan la decisión.

Un modelo de planificación jerárquica se puede descomponer en una serie de estructuras básicas, que consideran un nivel tope y un nivel base. Las decisiones del nivel tope son obligatorias para el nivel base. Sin embargo, las decisiones del nivel tope deben involucrar el nivel base, para respetar los alcances y limitaciones que se dan allí.

\section{Flujos de comunicación:}

Analizando con más detalle las estructuras de comunicación entre el nivel tope y el nivel base, se distingue:

- La decisión que se toma en el nivel tope y que afecta el nivel base se llama instrucción.

- Para tomar la decisión, el nivel tope debe tener en cuenta características del nivel base. El flujo de información del nivel base al nivel tope, que comunica esta información se llama anticipación.

- El nivel base puede reaccionar a una decisión del nivel tope (negociar). Esto ocurre, cuando en el nivel base se opina que las decisiones del nivel tope no se pueden realizar. Este hecho se inicia por un flujo llamado reacción. El nivel tope considera la reacción y modifica adecuadamente la instrucción.

En el nivel base se implementa la decisión, poniendo en práctica la decisión tomada en el nivel tope. De acuerdo con la implementación en el sistema objeto la jerarquía decisoria recibe la retroalimentación que puede inducir otro ciclo decisorio.

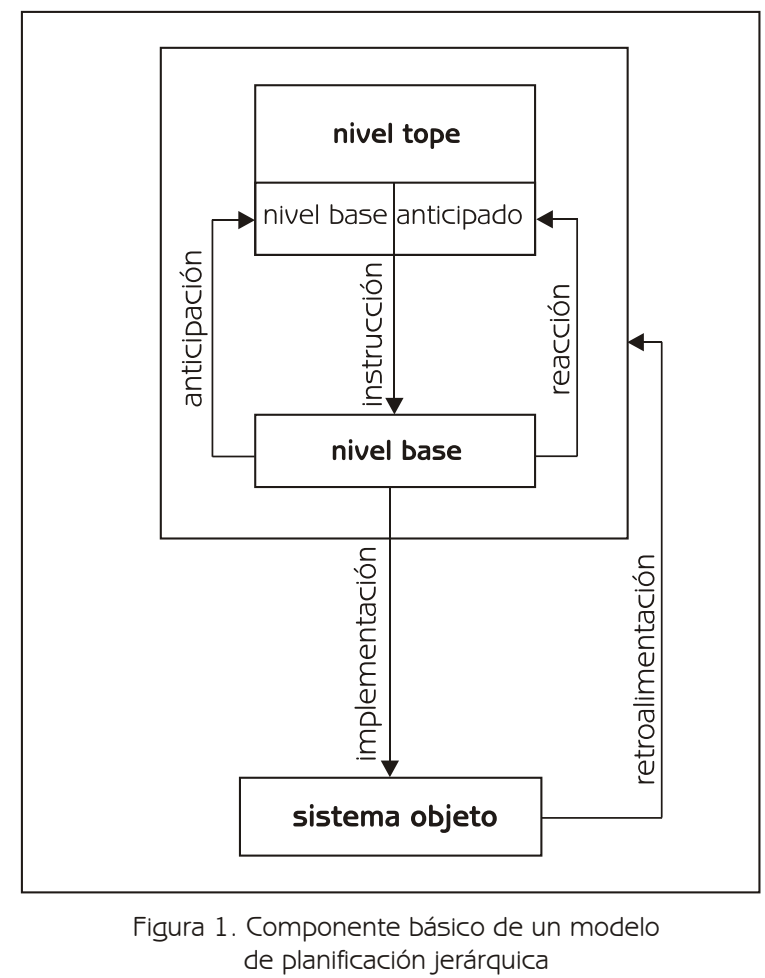

Agregación - desagregación de la información: El modelo jerárquico compone varias estructuras como las anteriores de acuerdo con la organización de la empresa, diferenciando por ejemplo entre los niveles estratégico, táctico y operativo (Figura 1). En cada nivel decisorio se considera la información en el grado de detalle que allí se requiere. En los niveles superiores se usa la información muy agregada, en los niveles bajos se consideran los detalles

La agregación y desagregación se aplica en todo tipo de información requerida para la planeación:

- El horizonte y los períodos de planeación: en el nivel estratégico se planea para horizontes largos, por ejemplo para un año en unidades de un mes. A los niveles inferiores se desagrega el horizonte, llegando en el nivel operativo por ejemplo a un horizonte de una semana 
detallado para períodos de un día.

- Los productos entran en la planeación en forma de familias, tipos o unidades de acuerdo con el nivel.

- Como recursos se pueden considerar en forma muy agregada las líneas de producción que se pueden desagregar obteniendo celdas productivas o recursos unitarios.

\section{La modelación en la planificación jerárquica:}

La jerarquía decisoria es la base para la modelación.

Se usa una serie modelos, de acuerdo con los niveles decisorios. Los modelos de decisión en cada nivel buscan alcanzar u optimizar sus propios objetivos. Para usar la información adecuadamente agregada, se puede disminuir el número de variables involucradas, llegando a modelos más sencillos y comprensibles y, por ende, más fáciles en el manejo computacional y en la interpretación.

Los modelos en cada nivel buscan alcanzar u optimizar sus propios objetivos; el logro de los objetivos del nivel superior se garantiza integrando estos como restricciones del modelo.

Inherente a la planificación jerárquica es el problema de factibilidad: ¿Es posible desagregar las decisiones tomadas en los niveles altos de tal forma que se obtenga una solución factible para el nivel inferior, consistente con la solución del nivel superior?

El problema de la desagregación factible se vuelve más complejo en situaciones de incertidumbre (Axäter et al., [1]). Si la demanda es estocástica con una distribución conocida, el problema desagregación consiste en maximizar la probabilidad de una solución factible en el nivel inferior. Los modelos estocásticos, que resuelven este tipo de problema, son computacionalmente más exigentes, como por ejemplo la programación dinámica que propone Axäter. Por eso se recomienda en casos de aplicación analizar si se puede usar un modelo determinista, basado en promedios o si es necesario usar un modelo estocástico, que se ajusta mejor a la realidad.

El sistema de apoyo a las decisiones jerárquico: Existen muchas investigaciones que se ocupan del desarrollo de sistemas de ayuda a las decisiones (DSS), pero pocas con la visión de integración empresarial de un sistema jerárquico. En Vicens [7] se ubican los conceptos de planificación jerárquica en el contexto de la integración empresarial, que supone un enfoque holístico del sistema productivo: Todos los componentes del sistema contribuyen para alcanzar la misión de la empresa. La integración empresarial implica la definición de un sistema de información integrado, que reflejando la estructura jerárquica de la empresa, permita el apoyo de las decisiones en todos los niveles de la organización.

EI DSS jerárquico se debe estructurar de acuerdo con la jerarquía, considerando para cada nivel una base de datos y un banco de modelos, los cuales están enlazadosjerárquicamente.

\section{CONTEXTO: EL INGENIO COLOMBIANO}

\section{Importancia del sector azucarero:}

El cultivo de la caña de azúcar es de mucha importancia en América Latina y el Caribe, y especialmente en Colombia, donde las condiciones de clima y suelo permiten el cultivo y la cosecha durante todo el año. Con su alta productividad de 1 tonelada de azúcar por hectárea y mes y un rendimiento de $11.6 \%$ de azúcar por peso de caña, la industria azucarera colombiana se destaca en el ámbito mundial. La producción de azúcar es una actividad tradicional en Colombia con un alto impacto socioeconómico en cuanto a generación de empleos y desarrollo de la infraestructura. El sector 
azucarero se puede considerar el generador principal de devisas en el país (por encima del café), con superávit de casi 300 millones US\$ (1998).

El sector pasó recientemente por una situación de crisis. La razón principal es el precio del azúcar que bajó de 30 US\$ centavos por libra en el año 1980 a un valor mínimo de 8 centavos en el 2000, recuperándose lentamente hasta quedar actualmente en 12 centavos. En contraste: los costos de producción de una libra oscilan alrededor de 8 centavos por libra de azúcar .

El presente trabajo se centra en la cosecha, el subsistema más complejo del ingenio azucarero, donde más problemas de planificación se presentan: una oferta escasa de caña ya que en el momento se aprovecha casi todo el área apropiada disponible para el cultivo, la influencia del clima que obstaculiza en las épocas de lluvia a todos los proceso de cosecha y la cantidad de recursos y tareas que deben ser asignados adecuadamente en el proceso de planificación como veremos con más detalle cuando hablemos del contexto de la investigación.

\section{La cosecha de la caña:}

El departamento de cosecha maneja toda la caña que luego se procesa en fábrica, tanto los cultivos propios de ingenio (alrededor del $30 \%$ ), como los de los proveedores externos. Las decisiones referentes a la cosecha no las toman los proveedores, sino el mismo ingenio que establece la fecha y el método de la labor, buscando el consenso con el proveedor, quien de esta forma está muy integrado en el sistema productivo.

El departamento de cosecha es responsable del suministro adecuado de caña a la fábrica. Los procesos principales de la cosecha son en su orden secuencial: la maduración, la quema, el

${ }^{1}$ Los datos estadísticos anteriores se encuentran en las páginas web de las organizaciones principales de la industria azucarera: CEMICAÑA, ASOCAÑA, TECMICAÑA. corte, el alce y el transporte de la caña. La maduración anticipada de la caña por aplicación aérea de un producto químico estimula el desarrollo de sacarosa en la planta. Alrededor de 6 semanas después de la aplicación del madurante, se alcanza el máximo contenido de sacarosa. Tradicionalmente, se quema el cultivo de caña antes del corte donde sea posible, para controlar plagas y sobre todo para eliminar las hojas secas, facilitando el corte y reduciendo el porcentaje de materia no aprovechable en la cosecha. El corte de la caña es casi siempre manual aunque existe también la posibilidad del corte mecánico. Es el proceso más intenso en cuanto al recurso humano. Un ingenio puede ocupar alrededor de 2.000 corteros adicional al personal requerido para la organización y supervisión del corte. Un cortero alcanza a cortar entre 7 y 12 toneladas de caña quemada en una jornada, su rendimiento en caña verde es más bajo. El proceso de alce de la caña es responsable para cargar la caña cortada en los equipos de transportes. Las máquinas alzadoras son el cuello de botella en los procesos de cosecha. El sistema de transporte de caña es el más susceptible a variaciones, siendo uno de los problemas de la cosecha la asignación y reasignación de los equipos de transporte. El personal y los recursos de alce y transporte están agrupados en frentes de cosecha, ya que trabajan juntos. Según el tamaño del ingenio se manejan entre 2 y 7 frentes de cosecha.

\section{EL MODELO GENERAL DE PLANIFICACIÓN JERÁRQUICA DE LA COSECHA}

\section{Los niveles decisorios:}

El modelo propuesto, basado en la estructura organizacional existente en el ingenio, define tres niveles decisorios: El nivel estratégico está representado por la gerencia, donde se toman las decisiones con una visión amplia. El nivel táctico se relaciona con los departamentos. En nuestro caso, con el de cosecha, las decisiones se definen a mediano plazo. El nivel operativo está representado por los responsables de los 
subprocesos, sus decisiones se toman a corto plazo (Figura 2).

\section{Los objetivos en los diferentes niveles:}

Las decisiones a tomar deben orientarse en los objetivos del ingenio, los cuales, en el problema de la planificación de la cosecha, se pueden especificar como en la figura 2. A lo largo del proceso de planificación en los diferentes niveles se debe tener en cuenta no sólo el objetivo de cada nivel, sino que también deben respetarse los objetivos de los niveles superiores, es decir, éstos restringen las decisiones en los niveles más bajos de manera que se puede hablar también de una jerarquía de los objetivos.

\section{Los niveles de agregación:}

En el modelo de planificación propuesto se definen diferentes grados de agregación de acuerdo con sus tres niveles decisorios. Se considera agregación del tiempo, agregación de los recursos, agregación de los procesos, agregación en la definición del origen de la materia prima y en el alcance de los objetivos.

El nivel estratégico con el grado más alto de agregación, considera sólo dos procesos macro: la cosecha y el procesamiento de la caña. Los recursos se definen como la fábrica y su capacidad de procesamiento y la cosecha con su capacidad total. Mo se diferencia el origen de la caña, se considera todo el terreno del ingenio. La planificación se hace a un año en períodos de un mes, lo que permite manejar diferencias de la capacidad de la cosecha a lo largo del año.

En el nivel táctico, los recursos se agregan a los frentes de cosecha. Para cada frente se definen dos procesos: las operaciones en campo y el desplazamiento entre diferentes lugares de trabajo. Además se considera el proceso de maduración, ya que las decisiones tácticas incluyen la selección de las suertes a madurar. A este nivel se consideran las suertes como origen de la caña. El horizonte de planificación es de tres meses para poder incluir la maduración con períodos de semanas para ello, días para los frentes.
El nivel operativo considera los procesos principales de cosecha: quema, corte, alce y transporte con sus recursos respectivos. Además se incluye el proceso de molienda. El horizonte para el nivel operativo es de quince días o hasta la próxima parada programada de la fábrica. Como período de planificación se define lo adecuado para cada proceso (para la quema y el corte: 1 día; para molienda, alce y transporte son turnos). Como origen de la caña se considera un área parcial de la suerte, donde se encuentra el frente.

En la ejecución de la cosecha interfieren muchos incidentes que obligan a ajustes de los programas. El modelo jerárquico para la planificación de la cosecha se entiende por esta razón como un modelo dinámico. El punto de partida siempre es el estado del sistema en el presente. Todas las proyecciones, la estimación de cada parámetro se basa en información actual.

\section{Los flujos de información:}

El nivel estratégico genera y transmite como instrucción el programa de producción a largo plazo que define para cada mes la cantidad de caña a cosechar y procesar. Se expresa en términos de toneladas de caña por día de trabajo. Este programa sincroniza entre la cosecha y la fábrica. Debido al proceso decisorio, este programa es factible, es decir se puede realizar con los recursos existentes en el ingenio bajo las condiciones esperadas cada mes.

En el nivel táctico se generan el programa de cosecha a medio plazo y el programa de maduración. El programa de cosecha a medio plazo fija para cada frente de cosecha el itinerario: Define las operaciones en campo (la suerte donde se ubica el frente, el tiempo de permanencia del frente en esta suerte y la cantidad de caña a cosechar diariamente) y el desplazamiento (origen y destino del desplazamiento, duración). La compatibilidad entre los programas a largo y a medio plazo se logra manteniendo la fecha de cosecha establecida por el programa a largo plazo, dentro 
de un rango de variación, definido también a nivel estratégico. El programa de maduración se deduce del programa de cosecha, definiendo la fecha de maduración que anticipe la fecha de cosecha por el período especificado y agrupando los resultados semanalmente. Los programas de este nivel se basan en el programa a largo plazo, respetando la cantidad total de caña a cosechar y procesar en cada mes.

Los programas a corto plazo son operativos. Se definen para cada uno de los procesos de cosecha. Se basan en un programa sincronizado entre el proceso de alce / transporte y la molienda, ya que estos son los procesos que limitan a los demás del programa de producción a corto plazo. Explícitamente se debe definir los programas: Quemas (define el área a quemar cada día); Corte (número de corteros y su localización); Alce (por frente: suerte y cantidad de caña a alzar y transportar); Transporte (número de equipos en cada suerte); Molienda (limita la capacidad al sincronizar con cosecha).

Los flujos de información de tipo anticipación se refieren a la información que emite desde un nivel inferior a un nivel superior para que éste derive la información requerida para la toma de decisiones. En el caso de la planificación de la cosecha esta información se refiere principalmente a la capacidad de los recursos, información que por agregación apropiada define los parámetros correspondientes del nivel superior.

Importantes para el modelo decisorio propuesto son también los flujos de reacción, por los cuales un nivel inferior informa sobre el cumplimiento o no de la instrucción. En nuestro caso se informa si en algún momento no se puede cumplir con lo programado. En los niveles superiores conduce este hecho a una reconsideración de la planificación.

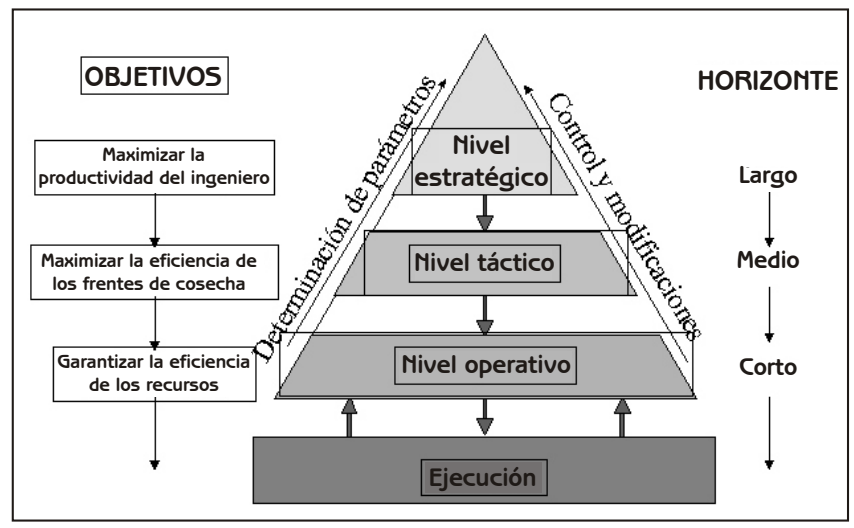

Figura 2. planificación jerárquica de la cosecha

\section{LA MODELACIÓN}

\section{La programación a largo plazo}

Por la programación a largo plazo se fija la cantidad de caña a cosechar y procesar mes a mes durante un año. Debe estar muy cercano a un objetivo importante de la empresa: maximizar la productividad. Sorprendente es que los ingenios parecen aplicar un indicador para la productividad que no es el más adecuado: Basan sus decisiones en el tonelaje de caña, es decir buscan procesar la máxima cantidad de caña posible. Es razonable planear las operaciones basado en el tonelaje de caña, pero al procesar la máxima cantidad posible de caña, no se optimiza necesariamente la cantidad de azúcar que el ingenio produce, siendo esta última una medida razonable para la productividad del ingenio.

Por ende se debe usar para la cosecha un indicador de productividad que contemple sacarosa contenida en la caña. Se introdujo el indicador TSH (toneladas de sacarosa por hectárea), que para la optimización de la productividad parece más indicado que el indicador actual TCH (toneladas de caña por hectárea). El indicador usado, afecta obviamente la edad de cosecha que se considera óptima. La figura 3 muestra los resultados de un modelo de simulación basado en datos reales que determina la cosecha anual en términos de caña y de sacarosa al aplicar diferentes edades de corte. 


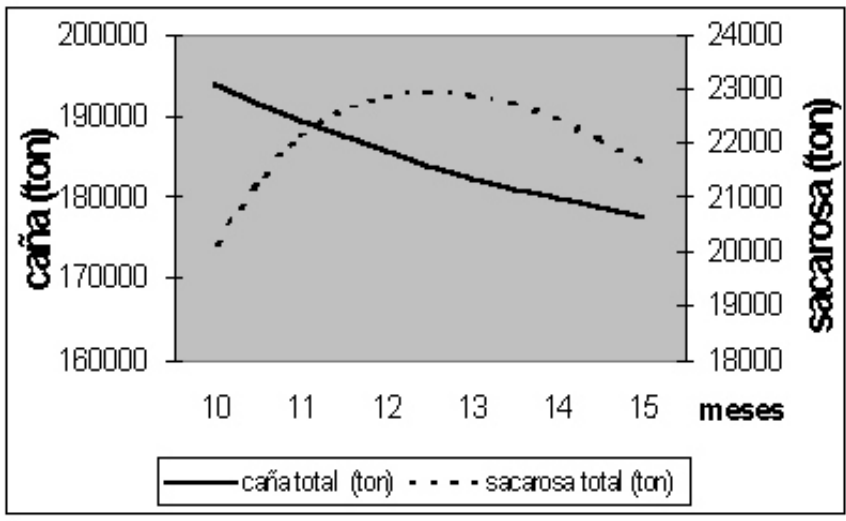

Figura 3. El tonelaje de caña y sacarosa en dependencia de la edad

Es evidente que la estrategia basada en el tonelaje de caña no es óptima: cosechando lo más temprano posible, se cuenta con más caña, pero la cantidad total de sacarosa es la mínima en el rango de edades del análisis. Claramente se manifiesta la superioridad de la estrategia basada en el contenido de sacarosa: cosechando a los 12 meses, se obtiene el máximo de sacarosa, aunque se manipula menos cantidad de caña.

Una vez determinado el indicador adecuado, se define el modelo para la planificación a largo plazo, como un modelo de optimización:

\begin{tabular}{|l|}
\hline \multicolumn{1}{|c|}{ Optimización de la Productividad del Ingenio } \\
\hline $\begin{array}{l}\text { Maximizar la cantidad de sacarosa total en la caña } \\
\text { Sujeto a las resctricciones de capacidad de cosecha y fábrica }\end{array}$ \\
\hline
\end{tabular}

La función objetivo es muy compleja, dado que implica determinar la sacarosa en la caña de todos los cultivos en dependencia de la edad de corte. Por esta razón se utiliza una heurística para determinar la solución del modelo. La heurística supone como solución inicial (no factible), que se coseche cada cultivo de caña en su fecha óptima en cuanto a sacarosa. Luego, se anticipe o posponga la cosecha, de acuerdo con las restricciones de capacidad de cosecha y fábrica. Cada cambio de cosecha implica un ajuste del tonelaje de caña y azúcar del cultivo correspondiente.

\section{La programación a medio plazo:}

La planificación a medio plazo propuesta tiene el propósito de maximizar la eficiencia de los recursos de cosecha (agregados en este nivel a frentes de cosecha). Consta de dos etapas principales: la distribución del terreno entre los frentes de cosecha y definir el orden en que se cosechan las suertes de cada frente.

Para la distribución en el espacio se supone que el terreno que maneja el ingenio, está repartido en zonas asignadas a los diferentes frentes de cosecha. El fin de esta distribución de terreno es mantener los frentes centrados en una zona determinada de trabajo para facilitar la programación de las operaciones de cosecha. En cada zona se define una parte central, donde sólo puede operar el frente asignado y una zona intermedia donde puede entrar un frente vecino. El modelo que reparte la caña de acuerdo a las zonas es un modelo de transporte tradicional:

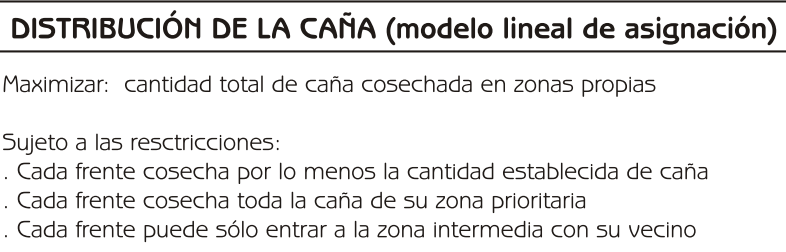

Si el modelo no tiene ninguna solución factible, la definición de las zonas intermedias no es apropiada, se deben ampliar.

Para definir el orden en que se cosechan las suertes de cada frente, se busca aplicar un algoritmo de secuenciación que optimice el recorrido. Esto significa que se tiene que modificar la fecha de cosecha de las suertes. Para controlar las modificaciones se usan ventanas de tiempo, que definen el período en el cual se puede cosechar una suerte. Al pasar de una fecha de cosecha a un período de cosecha, se gana la flexibilidad requerida para poder optimizar el recorrido.

El modelo busca minimizar el tiempo total de desplazamiento bajo las restricciones impuestas por las ventanas de tiempo. Entre más amplias se definen las ventanas, más flexibilidad hay en la 
desviación de la fecha óptima de cosecha (con el efecto negativo a la sacarosa), pero el tiempo de desplazamiento se reduce. Ventanas estrechas mantienen la cosecha cerca del óptimo, pero incurriendo en tiempos altos de desplazamiento. El modelo busca minimizar el tiempo total de desplazamiento.

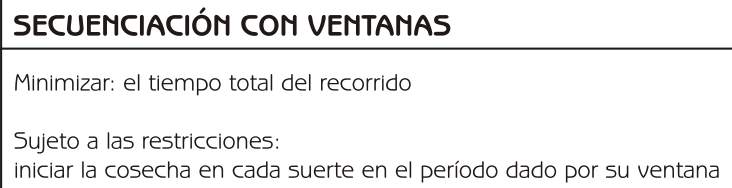

Los problemas de secuenciación se clasifican como MP-difíciles, es decir, el número de posibles soluciones crece exponencialmente con el número de nodos. En la situación de la cosecha tenemos que secuenciar entre 50 y 100 suertes, razón por la cual se debe usar un algoritmo heurístico.

Los algoritmos heurísticos parten de una solución que se mejora de forma iterativa. Problemático es evitar que la búsqueda quede atrapada en un mínimo local, que puede estar lejos de la solución óptima.

El algoritmo seleccionado para la solución del modelo es la Búsqueda local guiada (guided local search GLS) definido en Voudouris et al. [8]. Es un algoritmo iterativo que parte de un recorrido inicial. Se aplican de forma sistemática cambios al recorrido con el fin de obtener un recorrido más rápido. Para no quedar en un mínimo local, se agrega un término de penalización a la función objetivo, que penaliza los arcos de coste más bajo en la función objetivo, dando a otros arcos la posibilidad de formar parte de la solución.

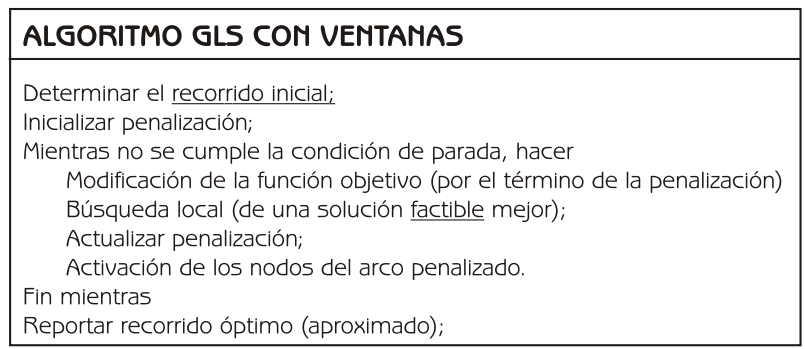

El algoritmo original no incluye las restricciones impuestas por las ventanas, razón por lo cual se debe adaptar el algoritmo en los siguientes sentidos (Tischer [6], Díaz [2]):

1. Determinar un recorrido factible inicial. Se usa un procedimiento análogo al "Método de dos fases" de optimización lineal: en una primera fase se aplica el algoritmo inicial con otra función objetivo, que busca minimizar la desviación de las ventanas. Si la desviación mínima es igual a cero, se logró una solución factible inicial, si no, no hay solución factible, y se debe suavizar las restricciones de las ventanas. En la segunda fase se parte de la solución factible para optimizar la función objetivo original.

2. Controlar el proceso de búsqueda para mantener la factibilidad. Sólo soluciones factibles mejores reemplazan la solución actual.

El algoritmo resultante se validó en dos aspectos: La cercanía de la solución obtenida a la óptima, obtenida por el algoritmo definido por Dumas et al, [3] y su aplicabilidad y utilidad en el problema de la secuenciación de la cosecha. En ambos sentidos, los resultados han sido muy satisfactorios: aplicado a problemas con datos sintéticos de una dimensión que permite usar el algoritmo exacto, el GLS arroja resultados muy cerca del óptimo en un tiempo de computación muy corto (menos de 2 segundos de CPU). En problemas con datos reales muestra que es capaz de mejorar mucho los tiempos de desplazamiento en comparación con los actuales (reducciones entre $25 \%$ y $40 \%$ ).

\section{La programación a corto plazo}

El problema esencial de la programación a corto plazo es la asignación adecuada de los equipos de transporte a los frentes de cosecha, que están operando en las diferentes suertes. Los equipos de transporte significan un recurso escaso, razón por la cual se deben distribuir de tal forma, que los procesos de cosecha y procesamiento serán lo menos obstaculizados posible. 
La Planificación Jerárquica y su Aplicación a la Cosecha de la Caña de Azúcar en Colombia

Como modelo se usó un modelo de colas con población finita, donde los frentes de cosecha representan los servidores, los equipos de transporte corresponden a los clientes. Como las distribuciones de las variables esenciales (tiempos de llegada, tiempos de servicio) no son completamente conocidas en la aplicación real, se decidió usar un modelo de simulación. Los escenarios del modelo de simulación son las posibles asignaciones. Como criterio de decisión se usó la cantidad total de caña transportada y el tiempo total que los frentes de cosecha esperan a un equipo de transporte.

Como resultado se obtiene que en algunos de los ingenios hacen falta más equipos de transporte para el desempeño adecuado de la cosecha, hecho que no quedó tan claro antes de usar el modelo y de mostrar por la simulación las deficiencias de la asignación actual.

\section{Validación del modelo de planificación:}

Para poder evaluar en forma realista la interacción entre los diferentes niveles decisorios, se determinó simular un ingenio con un grado muy alto de detalle. Se procedió de la siguiente manera:

Software: se usó Matemática (Wolfram Research Inc.), versiones 3.0 y 4.0, aprovechando las estructuras y operaciones de listas de este software, que facilita el manejo de datos y sus numerosas primitivas que simplifican el cálculo analístico y numérico.

Definición de los datos básicos: El ingenio virtual corresponde a un ingenio colombiano típico de tamaño entre medio y grande. La información detallada usada incluye: el terreno donde se ubica el ingenio, el área cultivada y su distribución; especificación de las suertes, variedades de caña (parámetros de desarrollo de biomasa y de sacaros) y su crecimiento; los recursos y su capacidad. Se definen los parámetros que se requieren para el proceso de planificación, básicamente la información que se supone conocida por anticipación en el modelo de planificación al iniciar el proceso decisorio.

Los modelos cuantitativos:

Se implantan los modelos cuantitativos que forman parte de los procesos decisorios en los diferentes niveles. Los parámetros de los modelos cuantitativos se determinan usando los datos básicos.

Simulación de los procesos decisorios:

Se simulan los procesos decisorios correspondientes a los diferentes niveles decisorios, suponiendo estrategias determinadas de planificación, para poder contrastar las propuestas del presente trabajo con la manera tradicional de tomar las decisiones. La simulación se lleva a cabo sucesivamente de los niveles altos a los niveles bajos, generando la información requerida por agregación de los datos básicos y aplicando los modelos cuantitativos y sus algoritmos de solución.

Los resultados de la validación por simulación son:

- Eficiencia del proceso decisorio: la toma de decisiones, basado en el modelo de planificación propuesto y alimentado con la información de la base de datos jerárquica, es rápida y eficiente.

- Modelación y algoritmos: Los modelos definidos para el apoyo del proceso decisorio arrojan los resultados adecuados en su contexto, comprobando la validez de la modelación. Los algoritmos implantados son eficaces y eficientes.

- Compatibilidad entre niveles decisorios: Los ajustes en la fecha de cosecha de las suertes que se deben a través de la jerarquía decisoria son mínimos en términos de tonelaje de caña y sacarosa.

\section{CONCLUSIONES}

Se desarrolló el modelo de planificación jerárquica para la cosecha de la caña, como herramienta para la toma de decisiones en el ingenio azucarero colombiano. Se espera que el modelo beneficie el sector en los siguientes aspectos: 
- La agilidad de los procesos decisorios, ya que se pueden apoyar en un modelo formal que describe el procedimiento de la toma de decisiones y los modelos que la soportan.

- La compatibilidad de los objetivos en los diferentes niveles de la organización. El modelo jerárquico define una jerarquía de objetivos, que se conservan en el proceso de detallar las decisiones hasta llegar al nivel operativo. Así se garantiza que se conozcan y se cumplan los objetivos generales de la empresa también en los niveles tácticos y operativos.

- La optimización del sistema. Como el modelo propuesto integra los diferentes componentes de la empresa y los orienta hacia un objetivo común, se espera obtener un mejoramiento de todo el sistema. El objetivo general del sistema es la productividad, que la planificación jerárquica busca mantener en todos los niveles. Como consecuencia, se espera un aumento de la productividad al aplicar el modelo propuesto. Pero en el marco de este objetivo general, se busca además una mayor eficiencia y eficacia en los diferentes subsistemas y procesos lo que se debe reflejar en un mejoramiento general del sistema productivo.

- La optimización de las decisiones detalladas en el marco del modelo global. La definición de los modelos adecuados y sus algoritmos eficientes de solución facilita los procesos decisorios en los niveles tácticos y operativos $y$ conduce a un mejoramiento de los subsistemas correspondientes, en el marco de la optimización del sistema como un todo.

- La previsión de problemas. El proceso de planificación incluye necesariamente las proyecciones del sistema hacia el futuro permitiendo que se puede anticipar posibles dificultades y tomar las medidas correctivas a tiempo.

- Con la presente investigación se sentaron las bases para el diseño y la implementación de un sistema de soporte a las decisiones (DSS) acerca de la cosecha de la caña. El sistema previsto consiste de una base de datos y una base de modelos en cada nivel, relacionando los niveles a través de la agregación de información, descrita anteriormente. La interacción adecuada entre datos y modelos y la utilidad del sistema está comprobada por la simulación del ingenio virtual. En el momento se están implementando los módulos relacionados con la planeación a corto y mediano plazo.

\section{BIBLIOGRAFÍA}

[1] AXÄtER, Sven; Ari, E. Aysen. 1988. Disaggregation under uncertainty in hierarchical production planning. European Journal of Operational Research 35.

[2] Díaz, Rosa María. 2000. Propuesta de un modelo de optimización para la secuenciación de la cosecha de la caña de azúcar. Tesis de grado, Plan de Matemáticas, Universidad del Valle, Cali, Colombia.

[3] DUMAS, Yvan; DESROSIES, Jacques; GELIMAS, Eric; SOLOMON, Marius. 1995. An optical algorithm for the traveling salesman problem with time windows. Operation Research 43\#2.

[4] HAX, A.C.; GOLOVIM, J.J; 1975. Hierarchical Integration of Production Planning and Scheduling. In: Studies in Management Science, Vol. 1 Logistics. Morth Holland American Elsevier, Mew York, 1975.

[5] SCHMEEWEISS, Christoph; 1995. Hierarchical structures in organizations: A conceptual framework. European Journal of Operational Research 86.

[6] TISCHER Irene. 2001. La planificación jerárquica integral de la cosecha de caña de azúcar en Colombia. Tesis doctoral, Universidad Politécnica de Valencia; España.

[7] VICENS, SALORT, Eduardo. 1999. Modelos de planificación jerárquica en gestión de sistemas de operaciones, en un contexto de la Integración Empresarial. Su incidencia en los sistemas de Información y Ayuda a la toma de decisiones. Trabajo de investigación; Universidad Politécnica de Valencia; Alcoy, España.

[8] VOUDOURIS, Christos; TSAMG, Edward. 1999. Guided local search and its application to the traveling salesman problem. European Journal of Operational Research, vol. 113. 